\title{
Evaluación nutricional de diferentes ensilajes para alimentar conejos
}

\author{
Nutritional evaluation of different silages to feed rabbits
}

Rigoberto Villa R. ${ }^{1}$; Jaime Hurtado V. ${ }^{2}$

1. M.S.c. Biología Vegetal, Universidad del Quindío, Armenia, Colombia, rivilla@uniquindio.edu.co.

2. M.S.c. Medio Ambiente y Desarrollo Sustentable, Universidad del Quindío, Armenia, Colombia, jhurtadov@uniquindio.edu.co.

Citar: VILLA, R.; HURTADO, J. 2016. Evaluación nutricional de diferentes ensilajes para alimentar conejos. Rev. Cienc. Agr. 33(1): 76-83. doi:http://dx.doi.org/10.22267/rcia.163302.54.

Recibido: Septiembre 30 de $2015 . \quad$ Aceptado: Enero 21 de 2016.

\begin{abstract}
RESUMEN
En la Granja Experimental Bengala de la Universidad del Quindío, ubicada en el municipio de Filandia, Quindío, se evaluó el valor nutricional de diferentes ensilajes para alimentar conejos, (pasto imperial Axonopus scoparius (Flüggé) Kuhlm.) mezclado con botón de oro (Ranunculus acris L.), ramio (Boehmeria nívea L.) y morera (Morus alba L.). Se valoraron cinco grupos de conejos conformados por cinco animales cada uno. El grupo de conejos alimentado con ensilaje de pasto imperial y botón de oro, fueron los que obtuvieron la mayor ganancia de peso $141 \mathrm{~g} /$ semanal, mientras que los conejos alimentados con solo forrajes frescos, obtuvieron un peso promedio de $109 \mathrm{~g} /$ semanal. Las fuentes nutricionales que mejor responden en la alimentación de conejos en su orden fueron: ensilaje de botón de oro, ensilaje de morera y ensilaje de ramio.
\end{abstract}

Palabras clave: alimentación, leguminosas, forrajes, neo zelandés.

\begin{abstract}
On the Quindío's University experimental farm “Bengala”, located in the municipality of Filandia, Quindío, was carried out an assessment regarding to the weight increase of rabbits for meat, fed on fodder, (imperial grass (Axonopus scoparius (Flüggé) Kuhlm) mixed with meadow buttercup (Ranunculus acris L.), ramie (Boehmeria nivea L.) and morus/mulberry (Morus alba L.). A completely randomized design with five probing groups of rabbits consisting of five rabbits each was used. The group fed on imperial grass and meadow buttercup ensilage, had the highest increase of weight (141g/weekly). Whereas the group fed on simply fresh fodder had the lower mean weight $(109 \mathrm{~g} /$ weekly). Thereafter, the nutritional food sources which better response for rabbits were silages based on meadow buttercup, morus/mulberry and ramie.
\end{abstract}


Key words: feeding, legumes, fodder, New Zealand

\section{INTRODUCCIÓN}

La alimentación del conejo, como el de las otras especies de interés zootécnico, representa el rubro más elevado dentro de los costos de producción, siendo necesaria la búsqueda de nuevas fuentes alimenticias tendientes a reducirlo, sin descuidar los requerimientos nutricionales del animal en cada una de sus fases biológicas (gestación, lactancia, cría, recría). Así mismo existe una limitada información que incluya la respuesta de conejos en crecimiento a diferentes fuentes suplementarias tropicales, sus combinaciones y su impacto económico (Mbanya et al., 2005).

La búsqueda de alternativas de alimentación que consideren el uso de recursos locales, constituye un elemento importante en la generación de formas de producción adecuadas para el trópico (Nieves et al., 2002). El ensilaje es un método de preservación para el forraje húmedo y su objetivo, es la conservación del valor nutritivo del alimento durante el almacenamiento. Este proceso, permite almacenar alimento en tiempos de cosecha conservando calidad y palatabilidad, lo cual posibilita aumentar la carga animal por hectárea y sustituir o complementar concentrados (Ashbell et al., 1999).

La calidad del ensilaje, es afectada por la composición química de la materia a ensilar, el clima y los microorganismos empleados, entre otros. El ensilaje se almacena en silos que permiten mantener la condición anaerobia. Existen varios tipos, y la escogencia del apropiado depende del tipo de explotación pecuaria, recursos económicos disponibles y topografía del terreno entre otros.

El proceso del ensilaje no mejora la calidad del forraje solo conserva su valor nutricional, como los componentes energéticos y proteicos mediante procesos de fermentación manteniéndolo estable por mucho tiempo. El ensilaje es el producto final que se obtiene cuando se conserva un alimento mediante un proceso de fermentación anaeróbico en estado húmedo y el propósito de esta técnica es mantener su valor nutritivo original, con un mínimo de pérdidas de materia seca y sin que se formen productos tóxicos que puedan perjudicar las funciones productivas y la salud de los animales (García, 1991, Filippi, 2011).

Otra de las características del ensilaje es la preservación de los forrajes, según Ashbell et al. (1999), es una técnica conocida desde hace mucho tiempo y es muy popular en América del Norte y en Europa. El uso de esta tecnología en gran escala requiere una mínima inversión en construcciones y equipos, un plan de trabajo muy estricto y bien coordinado durante las diversas fases del ensilado, y un conocimiento del proceso de ensilaje más profundo que el requerido para la henificación. Además, para ensilar bajo condiciones tropicales se debe prestar especial atención a tres aspectos muy importantes, tales como la madurez de la planta, la cual depende de las condiciones climáticas. En las zonas cálidas, el tiempo necesario para llegar a la madurez, puede ser mucho más corto y los cambios pueden ocurrir más rápidamente que en climas templados. En tales casos, es más difícil poder controlar el momento óptimo para la cosecha, lo cual es un momento crítico en los cultivos de cereales en los últimos estadios de madurez.

El contenido correcto de materia seca de la planta antes del ensilado, es un factor importante para el éxito de la fermentación. Los imprevistos climáticos como sequías, lluvias o altas temperaturas, pueden dañar el cultivo y aumentar las pérdidas.

La estabilidad aeróbica, el ensilaje se puede deteriorar rápidamente, especialmente durante la fase de distribución bajo climas cálidos y húmedos, se reduce la calidad del forraje y se producen pérdidas. Las temperaturas altas, facilitan el desarrollo de mohos y la actividad de las levaduras durante todo el año, razón por la cual debe evitarse el ingreso de aire dentro del silo y asegurar un tritura- 
do fino, buena compactación y sellado. Al abrir el silo y retirar ensilaje, se debe cuidar que la extracción deje una pared muy plana con el mínimo de superficie expuesta al aire. La estabilidad aeróbica, debería ser una prueba rutinaria en zonas calientes (Ashbell et al., 1991). Con este sistema se obtiene un alimento de aceptable a buena calidad nutricional empleando una mezcla de alimentos ricos en carbohidratos fermentables junto con substrato proteico no fermentable (Aguilera et al., 2007).

El conejo, es un mamífero de mediano tamaño, pelo suave y corto, orejas largas y cola corta, es una especie fundamentalmente crepuscular y nocturna. Pertenece al orden: Lagomorpha, familia: Lepóridae, género: Oryctolagus, especie: Oryctolagus cuniculus L. (Echeverry, 2004). El conejo es un animal herbívoro, vivaz, su alimentación es variada e ingiere la comida a gran velocidad; es resistente a las condiciones ambientales adversas, pues sus mecanismos digestivos y su cobertura cutánea, le permiten hacer frente al frío, a la falta de alimentos y a otras condiciones adversas (Velázquez, 2007).

Su ansiedad permanente y su frecuente inquietud están en relación directa con la densidad poblacional en que se encuentre, causando graves repercusiones sobre su fisiología, como la inhibición de los procesos digestivos, la ovulación provocada en hembras o la esterilidad en los machos. Por lo anterior, se recomienda la explotación de los conejos en jaulas individuales (Roca, 2008).

Los conejos presentan algunas peculiaridades anatómicas (como el desarrollo de sus pabellones auriculares), fisiológicas (por ejemplo la cecotrofia y la capacidad de las hembras para la ovulación estimulada). Por medio del proceso de la cecotrofia el conejo obtiene un suplemento de vitaminas de complejo B, proteínas de calidad que representan parte de sus necesidades nutritivas. Él conejo aprovecha un gran porcentaje de alimentos fibrosos, cuya digestión sería factible de no existir este singular reciclaje (González et al., 2007).

El conejo, por su eficiencia reproductiva, su rusticidad, fácil alimentación y manejo se ha convertido en una seria e importante alternativa para el pequeño y mediano productor; ya que cada día adquiere más importancia como productor alimentario, pues su índice de conversión de alimentos es muy alto. Solamente se necesita de 2,5 a 3,5kg de alimento para producir un kilogramo de carne (Barbado, 2006).

A nivel mundial, la cantidad de razas existentes es variada, siendo poco más de 45 reconocidas por la Asociación Americana de Cunicultura. No todas se producen de manera comercial, solo unas cuantas han alcanzado un desarrollo económico interesante, de ahí que, por aspectos prácticos, se seleccionaron aquellas razas más comunes y de mayor uso en sistemas de explotación comercial a nivel tropical para ser estudiadas (González et al., 2007).

La raza neozelandés es considerada productora de carne, procede del cruzamiento de Blanco Americano y Angora. Es blanco totalmente, de ojos color rojo, brillante y expresivo; su cuerpo de longitud media, compacto, redondeado y con buen desarrollo muscular, su longitud aproximada es de $47 \mathrm{~cm}$ para machos y de $49,5 \mathrm{~cm}$ para la hembra (midiendo de la punta de la cola a la punta del hocico). Existen diferentes variedades de la raza nueva zelanda como son: negra, roja y blanca (Echeverry, 2004).

Los conejos de ésta raza, poseen grandes masas musculares, el lomo y la grupa son rellenas y redondeadas; el peso ideal del macho adulto es de $4,5 \mathrm{~kg}$ y el de la hembra de $5 \mathrm{~kg}$. Esta raza, se caracteriza por su habilidad materna, docilidad, precocidad y ser de buena calidad peletera (calidad de piel para manufactura de artículos tales como bolsos, carteras, guantes entre otros). Además, presenta un buen rendimiento en canal, 
adaptado a diferentes climas y son muy prolíficos (González et al., 2007). Bajo éste contexto la presente investigación busco, evaluar la condición nutricional de diferentes ensilajes y medir los aumentos de peso en conejos para carne desde el destete hasta el momento de la comercialización.

\section{MATERIALES Y MÉTODOS}

Localización. Esta investigación, se realizó en el municipio de Filandia, Quindío en la vereda Cruces en la "Granja Experimental Bengala" de propiedad de la Universidad del Quindío, la cual tiene una altitud de 2050 msnm., y una temperatura promedio de $18^{\circ} \mathrm{C}$. El promedio anual de lluvia es de $2800 \mathrm{~mm}$. La región presenta dos temporadas de altas lluvias de febrero a marzo con una precipitación media de $248 \mathrm{~mm}$ y noviembre a diciembre con promedio de $386 \mathrm{~mm}$.

Diseño de tratamientos. Para la elaboración de los ensilajes se siguió el método propuesto por Betancourt et al. (2005) y Cañete et al. (1998). Se utilizaron pasto imperial (70\%) Axonopus scoparius (Flüggé) Kuhlm, mezclado con diferentes forrajes (30\%), botón de oro Tithonia diversifolia (Hemsl.) A. Gray, morera (Morus alba L.) y ramio (Boehmeria nívea L. Gaudich). Se cosecharon cada uno de los forrajes a ensilar, posteriormente, se sometieron a una deshidratación en campo una vez obtenido un 30 o 40\% de pérdida de agua se realizó el picado del material entre 3 y $5 \mathrm{~cm}$, aproximadamente. Después de fragmentado el material forrajero se procedió a la mezcla de los mismos, adicionándoles melaza en una proporción de $1 \mathrm{Kg}$ por cada $50 \mathrm{Kg}$ de forrajes a ensilar. Estas mezclas se empacaron en bolsas negras en forma compacta para extraer el aire y mantenerlas bajo condiciones anaeróbicas, de tal forma que se favorezca una buena fermentación. Las bolsas de silos se almacenaron en estivas en un lugar limpio, seco y fresco.

Obtención de gazapos. Paralelo al trabajo de preparación de los ensilajes, se preñaron e iniciaron las gestaciones de las conejas de la raza Nueva Zelanda, para la obtención de los gazapos necesarios para adelantar el trabajo en campo. Los gazapos criados, tuvieron un periodo de lactancia de un mes aproximadamente.

Una vez preparados los diferentes ensilajes, y obtenido el número suficiente de gazapos, se establecieron los diferentes grupos de conejos para iniciar el proceso de alimentación.

Se conformaron grupos de cinco conejos de manera aleatoria; se instalaron en jaulas tipo batería debidamente tabuladas y acondicionadas. Para cada tratamiento, se tomaron los pesos iniciales a cada uno de los animales.

Variables evaluadas. Se evaluaron los siguientes aspectos del ensilaje: características físicas y organolépticas de color, olor, textura, sabor (palatabilidad), consistencia, y para cada uno de los ensilajes elaborados se realizó un análisis bromatológico, además fueron pesados semanalmente cada uno de los animales de los diferentes tratamientos con el fin de terminar la ganancia de peso por grupo y tipo de alimentación, para obtener la ganancia de peso por grupo y tipo de alimentación.

Diseño experimental. Se utilizó un diseño experimental completamente al azar con cinco (5) tratamientos o fuentes nutricionales (T1. Pasto imperial + botón de oro; T2. Pasto imperial + Ramio, T3. Pasto imperial + Morera, T4. Pasto imperial, T5. Forrajes frescos) y cinco (5) conejos de raza nueva Zelanda por tratamiento. Se registraron semanalmente los pesos de cada conejo por un tiempo aproximado de tres (3) meses, se evaluó la ganancia de peso (medido en gramos), dado por el promedio de los conejos alimentados con las cinco (5) fuentes nutricionales (tratamientos).

Por medio del Análisis de Varianza se probó las hipótesis: 
Al utilizar los diferentes tratamientos, con sus diferentes niveles de inclusión, en promedio los tratamientos se comportan igual.

Al rechazarse la hipótesis nula (Ho), se procedió con la aplicación de la prueba de Rango de Newman Keuls para comparar promedios (Steel et al., 1985).

\section{RESULTADOS Y DISCUSIÓN}

Se obtuvieron ensilajes maduros, los cuales físicamente mostraron las siguientes características: color café claro, olor agradable. El forraje conservó todos sus contornos definidos y no humedece las manos al ser comprimido dentro del puño (Betancourt et al., 2005).

Según los análisis bromatológicos (Tabla 1), realizados a los diferentes ensilajes se pudo diferenciar el componente proteico a partir de los asocios de forrajes. El porcentaje de humedad en los ensilajes oscila entre un 54 y 76\%. Según Betancourt et al. (2005) explica que los ensilajes deben aportar gran contenido de humedad, en este aspecto todos los ensilajes se encuentran en un rango óptimo de porcentaje de humedad (De La Raza, 2005).

El valor energético, el valor nitrogenado y la ingestibilidad de los ensilados, vienen determinados por la calidad del forraje en el momento de su recolección y de las alteraciones producidas en el mismo, ligadas a las técnicas de recolección, mane- jo y conservación. Si todo el proceso no se realiza adecuadamente, los resultados pueden ser negativos (de la Roza, 2005).

El contenido de cenizas para los distintos tratamientos, fluctuó entre 8,02 y 12,56\%; contenidos mayores a $12 \%$ de $\mathrm{Ce}$, son asociados a contaminación con suelo durante la cosecha o elaboración del ensilaje (Chaverra y Bernal, 2000), lo que favorece la presencia de fermentaciones secundarias y reducción del consumo, situación que no sucede en el presente trabajo.

Según Guzmán et al. (2012) las investigaciones en forrajes ensilados, el porcentaje de cenizas que presentan está entre el 5 y 10\%, condición que cumplen cada uno de los ensilajes. Para García (1991), las cenizas deseables en un ensilaje, se situarían en un máximo de $12 \%$ y como se puede observar el silo de pasto imperial y ramio supero dichoporcentaje.Enlaconservación porensilajes, se trata de inhibir el crecimiento de microorganismos (bacterias y hongos) degradadores de la materia seca (Garcés et al., 2006). Así mismo, contenidos mayores a $12 \%$ de cenizas en materiales ensilados a base de maíz, son asociados a contaminación con suelo durante la cosecha o elaboración del ensilaje lo que favorece la presencia de fermentaciones secundarias y reducción del consumo (Chaverra y Bernal, 2000).

En relación con el porcentaje de grasa en cualquier

Tabla 1. Análisis bromatológico para ensilajes elaborados a partir de pasto imperial con botón de oro, ramio y morera.

\begin{tabular}{lccccc}
\hline \multicolumn{1}{c}{ ANÁLISIS } & $\begin{array}{c}\text { Imperia I+ } \\
\text { Botón Oro }\end{array}$ & $\begin{array}{c}\text { Imperial + } \\
\text { Ramio }\end{array}$ & $\begin{array}{c}\text { Imperial + } \\
\text { Morera }\end{array}$ & $\begin{array}{c}\text { Pasto } \\
\text { Imperial }\end{array}$ & Método \\
\hline Humedad \% & 76,44 & $71,21 \%$ & $69,01 \%$ & $54,02 \%$ & AOAC 969,38 B \\
Cenizas $\%$ & 10,44 & $12,56 \%$ & $8,02 \%$ & $10,03 \%$ & AOAC 942,05 \\
Grasa \% & 0,73 & $0,82 \%$ & $1,02 \%$ & $1,07 \%$ & NTC 668 \\
Proteína* \% & 8,28 & $8,95 \%$ & $6,66 \%$ & $4,90 \%$ & AOAC 978,04 \\
\hline
\end{tabular}

*El porcentaje de proteína es en base seca, el factor utilizado es de 6,25 correspondiente a todos los otros alimentos. 
ensilaje, esta descrito por los ácidos grasos volátiles, los cuales son producto de los ácidos orgánicos, como el ácido láctico, acético, entre otros. Según esta investigación, la mayor concentración de grasas la tiene el pasto imperial (1,07\%). El valor mínimo de ácidos grasos que requiere un ensilaje agradable y catalogado como de buenas características, es de 1,5 a $2 \%$, mientras que un $6 \%$ se estima como muy malo (Garcés et al., 2011).

De igual forma, Wattiaux, M, 2005, con relación a los cambios en la fracción proteica (nitrógeno), afirman que el ensilado cambia la fracción de proteína de los forrajes. La respiración es responsable de la ruptura de la proteína. A medida que la planta muere luego de cortada, las enzimas proteoliticas se rompen en componentes más pequeños, solubles, incluyendo péptidos, aminoácidos (el bloque constructor de la proteína) y amoniaco. La mayor degradación de proteína que tiene lugar en un silo, ocurre las primeras 24 a 72 horas (fase 1 y fase2) de la fermentación del ensilaje).

Por medio del ensilaje, se puede almacenar y reservar el alimento para su uso posterior con pérdidas mínimas de calidad nutricional (Garcés et al., 2006; Qamar, 2009 y Reyes et al., 2009).
En la Figura 1, se puede observar el promedio de peso inicial, los promedios finales del aumento de peso y el diferencial de aumento en peso vivo en cada uno de los grupos alimentados con los diferentes ensilajes elaborados y con forrajes frescos.

Los grupos de conejos alimentados con ensilajes de especies forrajeras asociadas (Imperial+Boton de Oro, Imperial+Morera, e Imperial+Ramio), fueron los tratamientos con un mejor desempeño en aumento de peso (141, 137 y 134g); Garcia et al. (2009), utilizando un alimento comercial reportaron ganancias de peso de $37 \mathrm{~g} /$ día en gazapos neozelandés que consumían en promedio, 130g/día.

Deeste resultado, se desprenden dos valoraciones: en primer lugar, los ensilajes se convierten en una alternativa real para la alimentación de conejos dedicados a la producción de carne y segundo cuando estos ensilajes se elaboran a partir de asocios de forrajes (imperial con botón de oro, morera o ramio), se fortalecen los valores nutricionales de los mismos (Tabla 1), lo que

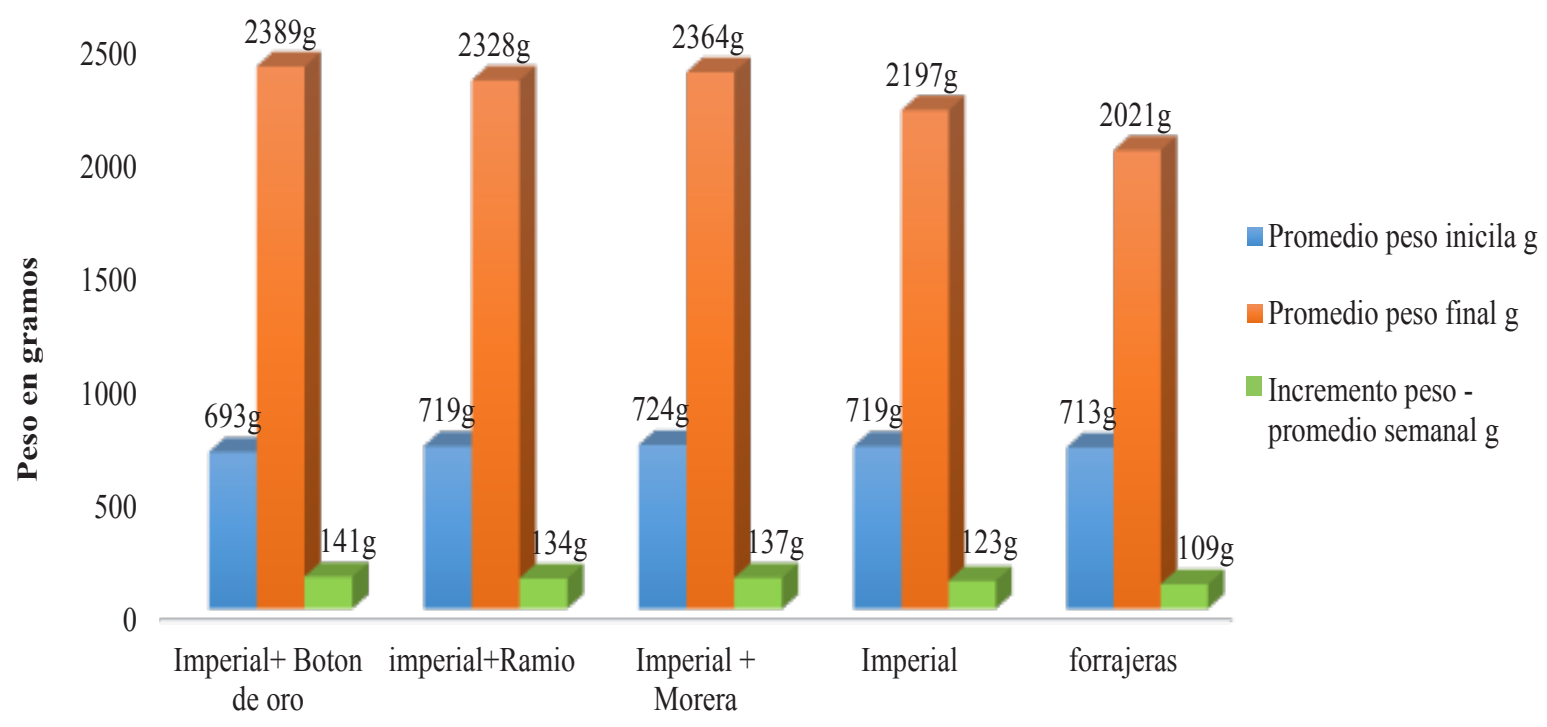

Figura 1. Peso promedio de conejos alimentados con diferentes ensilajes. 
redunda en un comportamiento más adecuado en la intención de producir carne en la empresa cunícola.

El análisis revelo que se presentan diferencias estadísticamente significativa $(\mathrm{p}<0,05)$, entre los grupos 1-5, 3-5 y 2-5, lo cual confirma que el grupo alimentado con ensilaje de pasto Imperial +Botón de Oro, fue el de mayor aumento en peso (141g). El botón de oro ha sido reconocida entre los productores como una planta con un importante valor nutricional, principalmente por su capacidad para la acumulación de nitrógeno (Medina et al., 2009, Verdecia et al., 2011) y por el nivel de fibra bruta, siendo éste del 31,6\% a los sesenta días de edad (Roa et al., 2010), características que dejan al botón de oro en condiciones nutricionales similares a las de otras plantas arbustivas destinadas a la producción forrajera.

\section{CONCLUSIONES}

Los conejos alimentados con ensilajes obtienen la mayor ganancia de peso, que los animales alimentados con solo forrajes frescos. El ensilaje de pasto Imperial+Botón de Oro, pueden mejorar la calidad de la dieta para alimentar conejos y así optimizar la productividad animal.

El mejor tratamiento en cuanto a ganancia de peso fue el ensilaje de pasto Imperial+Botón de Oro. Cuando los ensilajes son elaborados con asocios de gramíneas y forrajes se fortalece la ración alimenticia, lo que redunda en un mejor desempeño por parte del animal, en este caso una ganancia de peso.

\section{AGRADECIMIENTOS}

Vicerrectoría de Investigaciones de la Universidad del Quindío por su financiación.

\section{REFERENCIAS BIBLIOGRAFÍCAS}

AGUILERA, A.; PÉREZ, F.; GRANDE, D.; DE LA CRUZ, I.; JUÁREZ J. 2007. Digestibility and fermentative characteristics of mango, lemon and corn stover silages with or without addition of molasses and urea. Small Ruminant Research, 26:87 - 91.

ASHBELL, G.; WEINBERG, Z.G.; BOLSEN, K.K.; HEN, Y.; AZRIELI, A. 1999. The silage characteristics of two varieties of forage sorghum mixed in different proportions at two stages of maturity. African J. Range Forage Sci. 15:69 - 72.

ASHBELL, G.; WEINBERG, Z.G.; AZRIELI, A.; HEN, Y.; HOREV, B. 1991. A simple system to determine the aerobic determination of silages. Can. Agric. Engin. 33:391 - 395.

BARBAD0, J. L. 2006. Cría de conejos, su empresa de cunicultura. Ed. Albatros. Argentina. 190 p.

BETANCOURT, M.; GONZALEZ, I.; MARTINEZ DE ACURERO M. 2005. Evaluación de la calidad de los ensilajes. Revista Digital Centro Nacional de Investigaciones Agropecuarias de Venezuela. 8:1 - 5.

CAÑETE, M. V.; SACHA J. 1998. Ensilado de forrajes y su empleo en la alimentación de rumiantes. Ediciones Mundi-Prensa, Madrid, España. 1 - 260 p.

CHAVERRA, G.; BERNAL. E. 2000. Ensilaje en la alimentación de ganado vacuno. IICA. Tercer Mundo Editores. Bogotá, Colombia. 65 - 123 p.

DE LA RAZA, B. 2005. El ensilado en zonas húmedas y sus indicadores de calidad. IV Jornadas de Alimentación Animal. Laboratorio de Mouriscade. Lalín. 1 - 20 p.

ECHEVERRY J. 2004. Explotación y Manejo de Conejo Doméstico. Politécnico Colombiano Escuelas de Ciencias Agrarias. En: http://granjalagabriela. blogspot.com/2008/05/historia-de-la-cunicultura. htm; consulta: marzo, 2014.

FILIPPI, R.2011. Conceptos básicos en la elaboración de ensilajes. Universidad de la Frontera. Chile. 1 - 9 p.

GARCÍA, G.1991. Ensilado de Forrajes. Publicaciones de Extensión Agraria. Madrid España. 5 - 7 p.

GARCÍA, D., P. LARA, E. MAGAÑA, E. AGUILAR, J. SANGINES. 2009. Parámetros reproductivos en conejas alimentadas con morera (Morus alba) ó tulipán (Hibiscus rosa-sinensis). Revista Verde (Mossoró- RN Brasil). 4(3):90 - 98.

GARCÉS, M.; A.; SUÁREZ, E.; SERNA, J.; RUÍZ. S. 2006. 
Evaluación de la calidad bromatológica del ensilaje de pasto kikuyo y maní forrajero. Revista Lasallista de Investigación. 3(2):34 - 37.

GARCÉS, A.M.; BERRIO, L.; RUÍZ, S.; SERNA, J.; BUILES. A. 2011. Ensilaje como fuente de alimentación para el ganado. Rev. Lasallista Investigación. 1(001):66 - 71.

GONZÁLEZ, P., CARAVACA-RODRÍGUEZ F.P., 2007. Producción de conejos de aptitud cárnica. Sistemas ganaderos en el siglo XXI. Universidad Sevilla. España. 443 - $461 \mathrm{p}$.

GUZMÁN, 0.; LEMUS, C.; MARTÍNEZ, S.; BONILLA, J.; PLASENCIA, A.; LY, J. 2012. Chemical characteristics of silages of mango (Mangifera indica L.) by products for animal feeding. Cuban J. Agric. Sci. 46(4):369 - 374.

MBANYA, J. N., B. N. NDOPING, J. M. MAFENI, D. W.FOMUNYAM. 2005. The effect of different protein sources and their combination on the performance of growing. En: http://www.lrrd.org/lrrd17/3/ mban17032.htm; consulta: mayo, 2015.

MEDINA, M.; GARCÍA, D. E.; GONZÁLEZ, M.; COV, L, MORANTINOS, P. 2009. Variables morfo-estructurales y de calidad de la biomasa de Tithonia diversifolia en la etapa inicial de crecimiento. Zootecnia Trop. 27:121 $-134$.

NIEVES, D.; TERÁN, O.; SILVA, L.; GONZÁLEZ, C. 2002. Digestibilidad in vivo de nutrientes en dietas en forma de harina con niveles crecientes de Leucaena leucocephala para conejos de engorde. Rev. Científ. 12(2):408 - 411.

NISA, M.; SHAHZAD, M.A.; SARWAR, M.; TAUQIR, N.A. 2008. Influence of additives and fermentation periods on silage characteristics, chemical composition, and in situ digestion kinetics of Jambo silage and its fodder in Nili buffalo bulls. Turk. J. Vet. Anim. Sci. 32(2):67 - 72

QAMAR, M. 2009. Effect of molasses and corn as silage additives on the characteristics of Mott Dwarf Elephant grass silage at different fermentation periods. Pak. Vet. J. 29:19 - 23.

ROCA, T. 2008. Razas de Conejos. En: http://www. conejosinfo.com/artículos/razas-de-conejos; consulta: octubre, 2014.

REYES, N.; B. MENDIETA; T. FARINAS; M. MENA; J. CARDONA; D. PEZO. 2009. Elaboración y utilización de ensilajes en la alimentación del ganado bovino, Manual Técnico. Primera Edición. CATIE. Managua, Nicaragua. $98 \mathrm{p}$.

ROA, M., CASTILLO, C., TÉLLEZ, Y E. 2010. Influencia del tiempo de maduración en la calidad de ensilajes con forrajes arbóreos. Revista Sistemas de Producción Agroecológicos 1(1): 63-73.

STEEL, R.; TORRIE, J.; MARTÍNEZ, R. 1985. Bioestadística: Principios y Procedimientos. McGrawHill. Bogotá, Colombia. 622 p.

VERDECIA, D.; RAMÍREZ, J.; LEONARD, I.; ÁLVAREZ, Y.; BAZÁN, Y.; BODAS, R.; ANDRÉS, S.; ÁLVAREZ, J.; GIRÁLDEZ, F.; LÓPEZ. S. 2011. Calidad de la Tithonia diversifolia en una zona del Valle del Cauto. REDVET. En: http://www.veterinaria.org/revistas/redvet/ n050511/051113.pdf; consulta: noviembre, 2014.

WATTIAUX, M. 2005. Guía Técnica Lechera. Instituto Babcock para la investigación y desarrollo internacional de la industria lechera. 9 - $20 \mathrm{p}$. 